\title{
A Novel Design of a Miniature Metamaterial Antenna for RFID Reader Applications
}

\author{
A. Ennajih ${ }^{1}$, J. Zbitou ${ }^{2}$, M. Latrach ${ }^{3}$, A. Errkik ${ }^{4}$, L. El Abdellaoui ${ }^{5}$, A. Tajmouati $^{6}$ \\ $1,2,4,5,6$ LMEET FST of Settat, Hassan 1st University, Settat, Morocco \\ ${ }^{3}$ Microwave Group (ESEO), Angers, France \\ ${ }^{*}$ Corresponding author, e-mail: abdelhadi.ennajih@gmail.com
}

\begin{abstract}
In this article, we propose a novel design of a dual band antenna based metamaterial for RFID applications. The proposed antenna consists of a rectangular patch using inverted $u$-slot in radiating element and two split ring resonators loaded in the ground plane. The antenna is printed on an FR4 substrate having athickness of $1.6 \mathrm{~mm}$, permitivitty of 4.4 and loss tanget of 0.025 . this antenna is feed by a micostrip line having caracteristique of $50 \mathrm{Ohms}$. The designed antenna resonates at the UHF band around $900 \mathrm{Mz}$ with an input impedance of $87 \mathrm{MHz}$ and also, resonates at the ISM band around $2.45 \mathrm{GHz}$ with a large bandwidth about $516 \mathrm{MHz}$. The designed antenna is simulated bys using CST and the results is validated by HFSS. After simulation, a prototype of the proposed antenna is fabricated, and the measured results show a good agreement with the simulated one.
\end{abstract}

Keywords: radio frequency identification, metamaterial, RFID reader antenna, UHF antenna

Copyright $@ 2018$ Universitas Ahmad Dahlan. All rights reserved.

\section{Introduction}

RFID stands for radio frequency identification, this technologie uses radio waves to locate, track and identify animals, objects or people [1]. RFID technology has been rapidly developing in recent years; it can be applied in many service industries, distribution, discard goods management, animal control, security, logistic, manufacturing companies [2]. Several frequency bands have been allowed to the RFID technology, such as low-frequency LF (125 $\mathrm{KHz}-134 \mathrm{KHz})$, high-frequency $\mathrm{HF}(13.56 \mathrm{MHz})$, ultra-high frequency UHF $(860 \mathrm{MHz}-960 \mathrm{MHz})$ and microwave band MW $(2.45 \mathrm{GHz}$ or $5.8 \mathrm{GHz})$ [3]. However, the UHF band is preferred in many applications due to the merits of high data transfer rate broad readable range. In the UHF RFID band, each country has its own allocation. For example, 866-869 MHz in Europe, 902-928 $\mathrm{MHz}$ in North and South America, 950-956 MHz in Japan and some Asian countries [4].

An RFID system requires at least three components, a reader, a transponder, and database. The RFID tag or transponder is a simple device that can store unique identification information of tagged object. The RFID reader contains an RF transceiver module communicates with the tag via radio signals and the back-end database associate arbitrary records with the tag identifying data. Figure 1 illustrates the basic components of an RFID system.
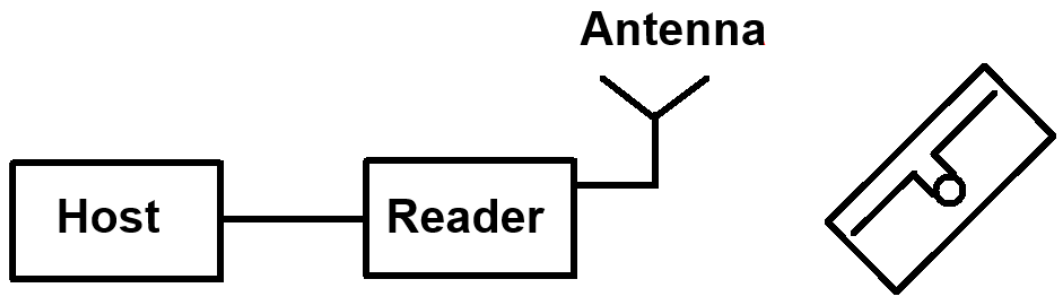

Tag

Figure 1. Basic components of an RFID system 
In this work, a monopole antenna by using $\mathrm{U}$-shaped slots in the radiation patch for UHF RFID applications based on metamaterial is investigated. Without inserting the split rings resonators (SRR), the monopole antenna operates at $920 \mathrm{MHz}$. The second band is achieved by adding the metamaterial unit cells in the ground plane. This paper is organized as follows, section II describes the design of metamaterial unit cell, section III presents the antenna design, section IV gives the simulated results and section $\mathrm{V}$ concludes this paper.

\section{Design of a Metamaterial Unit}

Metamaterial is artificial metallic structures having simultaneously negative permittivity and permeability, and consequently, have a negative index of refraction. It gains its properties from structure rather than composition [5]. the subject of metamaterials started with the Russian theorist Victor Veselago in 1967 [6]. Veselago introduced a new kind of material, which has a negative permittivity and a negative permeability. Then, Pendry and al. analised and proposed some structures wich can provid a negative permittivity and a negative permeability [7] [9]. They declared that we can obtain negative permittivity by using thin metallic wires [6] and negative permeability can be obtained by metallization of split rings resonators [8]. Inspired by the theoretical works of Pendry, Smith and his group had been firstly fabricated the first artificial metamaterial [9]. After these works, metamaterial has attracted a great attention of researchers in various devices such as antennas, filters, amplifiers and others [10-11]. In this paper, we have used a metamaterial unit cell formed by two rectangular split-ring resonators to operate at 2.45 GHz. The geometrical parameters are chosen by using the optimization technique integrated into CST Microwave Studio. After many series of optimization, the final dimensions of the metamaterial unit cell are as follows; the length, the width and the height of the outer ring are $L r=13 \mathrm{~mm}, W r=19 \mathrm{~mm}$, and $W r 1=1.5 \mathrm{~mm}$ respectively. The separation between the inner and the outer ring $g 1=2 \mathrm{~mm}$, the gap at the split of both rings $\mathrm{g}=2 \mathrm{~mm}$, and the distance between two SRR is $1 \mathrm{~mm}$. the geometry of the proposed metamaterial unit cell in a TEM waveguide is illustrated in Figure 2.

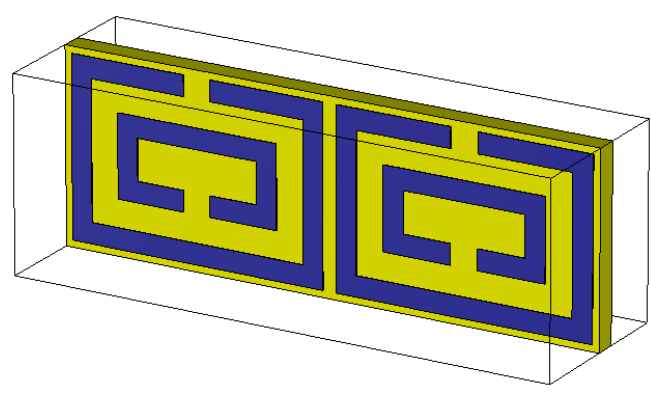

Figure 2. (a)The proposed metamaterial unit cell. (b)S-parameters of the proposed metamaterial unit cell

The effective permittivity and permeability are extracted by using the following equations [12]:

$$
\begin{gathered}
\mu_{e f f}=\frac{2}{J K_{0} d} \frac{1-V_{2}}{1+V_{2}} \\
\varepsilon_{\text {eff }}=\frac{2}{J K_{0} d} \frac{1-V_{1}}{1+V_{1}}
\end{gathered}
$$

Where $\mathrm{V} 1$ and $\mathrm{V} 2$ are calculated by the following equations:

$$
V_{1}=S_{21}+S_{11} \text { and } V_{2}=S_{21}-S_{11}
$$


The extracted parameters are illustrated in Figure 3. We can conclude from this figure that by using two metamaterial unit cells, a simultaneously negative region of permittivity permeability around $2.45 \mathrm{GHz}$ is obtained.
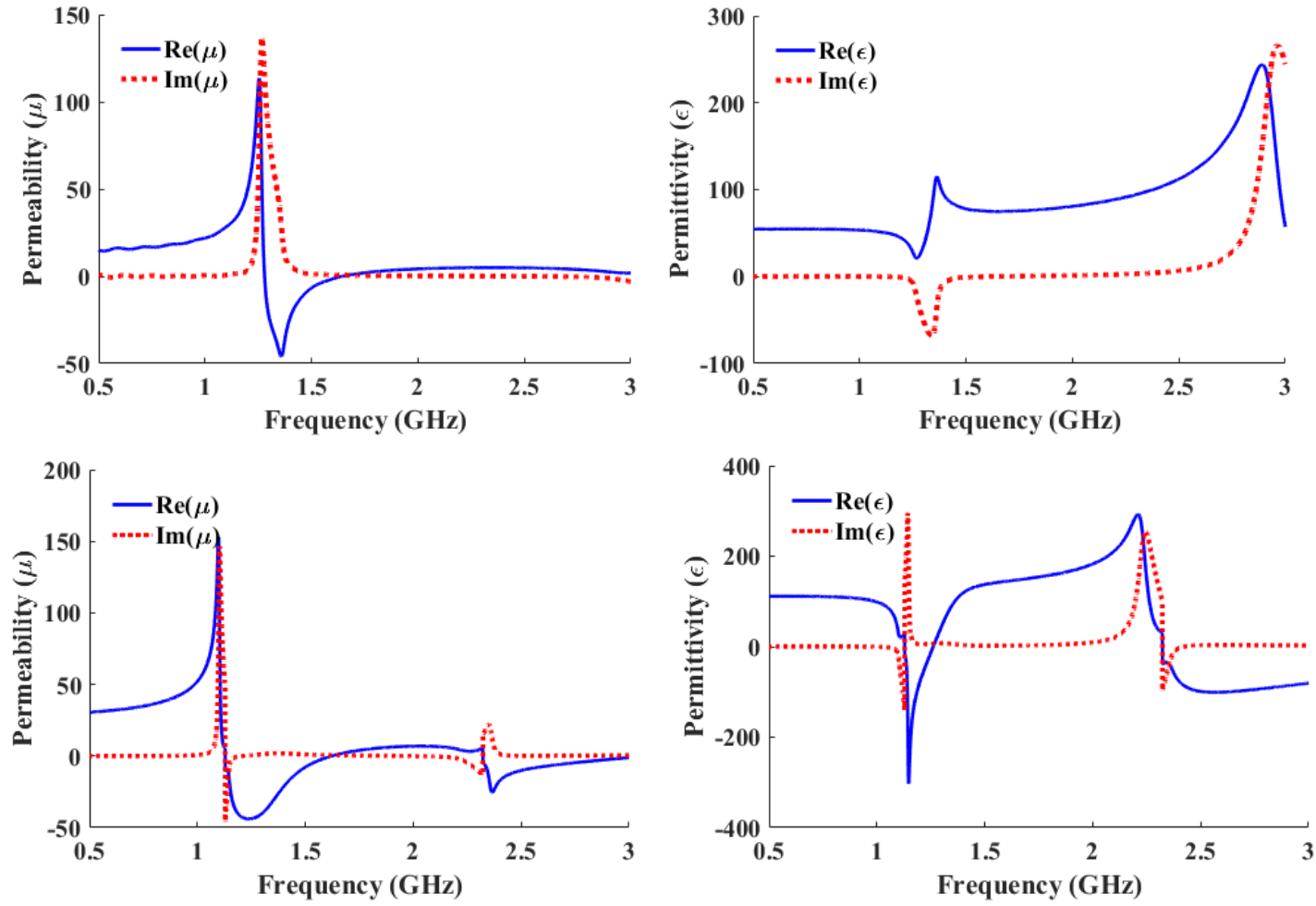

Figure 3. Permeability and permittivity of the proposed metamaterial unit cell

(a)1 SRR (b)2 SRRs

\section{Antenna Design}

The designed antenna is composed of a rectangular patch and a U-slot is inserted in the front side and two metamaterial unit cells formed by split ring resonators (SRR) are placed on the opposite side of the patch. The SRR is used to achieve the dual-band operation. The antenna is feed by a microstrip line having an impedance of 50 ohms. Figure 4 represents the geometry of the proposed antenna. The antenna is designed on an FR4 epoxy substrate having thickness of $1.6 \mathrm{~mm}$, a permittivity of 4.4 and, loss tangent of 0.025 . The proposed antenna with optimum dimensions has been simulated and optimized by using CST microwave studio. The total size of the proposed antenna is $85 \mathrm{~mm} \times 50 \mathrm{~mm}$. The optimum values of the structural parameters of the proposed antenna are illustrated in Table 1.

Table 1. Geometrical Parameters of The Proposed Antenna

\begin{tabular}{cccc}
\hline parameter & $\begin{array}{c}\text { Dimension } \\
(\mathrm{mm})\end{array}$ & parameter & $\begin{array}{c}\text { Dimension } \\
(\mathrm{mm})\end{array}$ \\
\hline $\mathrm{Wp}$ & 30 & $\mathrm{Lu}$ & 18 \\
$\mathrm{Lp}$ & 38 & $\mathrm{Wu1}$ & 2 \\
$\mathrm{Lf}$ & 45 & $\mathrm{Wg}$ & 50 \\
$\mathrm{Wu}$ & 18 & $\mathrm{Lg}$ & 7 \\
\hline
\end{tabular}




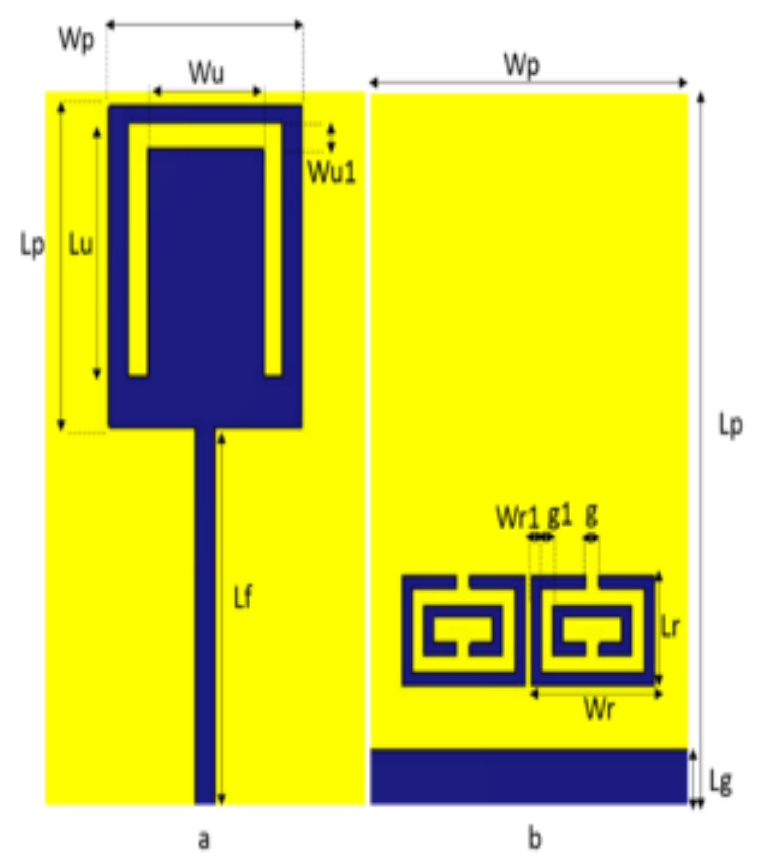

Figure 4. Geometry of the proposed antenna (a)front view (b)back view

\section{Results and Analysis}

Figure 5 presents the simulated reflection coefficient of the final geometry of the proposed monopole antenna with and without metamaterial. It can be observed that the antenna without SRRs operates at $925 \mathrm{MHz}$ with a return loss of $-24 \mathrm{~dB}$. However, by using metamaterial the antenna resonates at $900 \mathrm{MHz}$ with a return loss of $-33.37 \mathrm{~dB}$ and $2.45 \mathrm{GHz}$ with a return loss of $-38.43 \mathrm{~dB}$. The impedance bandwidth is $87 \mathrm{MHz}$ at the first frequency band and $516 \mathrm{MHz}$ at the second frequency band.

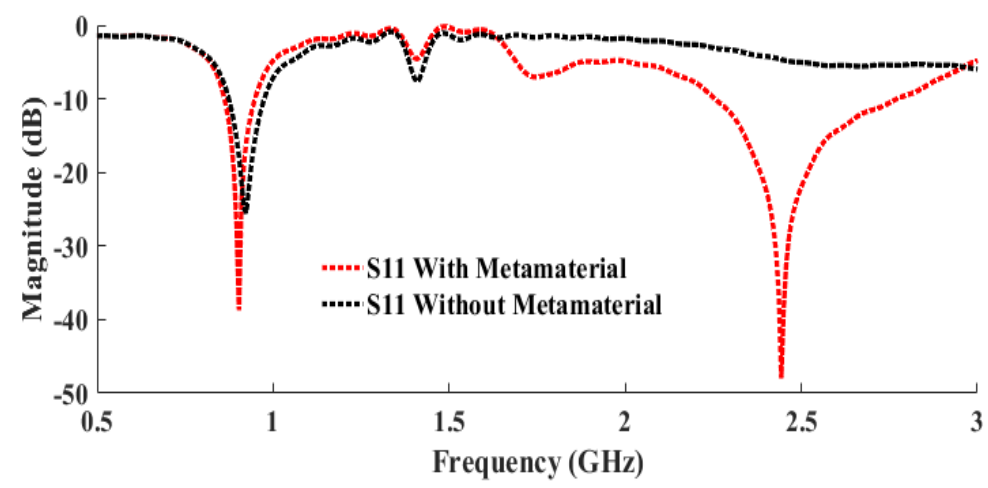

Figure 5. Simulated reflection coefficient of the proposed antenna with and without metamaterial

The simulated gain versus frequency of the proposed antenna is illustrated in Figure 6 . According to the figure, the peak gain is $2.2 \mathrm{~dB}$ at the first frequency band and $3.6 \mathrm{~dB}$ at the second frequency band.

Figures 7 and 8 illustrate the 2D radiation patterns at $2.45 \mathrm{GHz}$ and $900 \mathrm{MHz}$ at E-plane and $\mathrm{H}$-plane. The designed monopole metamaterial antenna has an omnidirectional radiation pattern at the UHF band and ISM band for both E-plane and $\mathrm{H}$-plane. The angular width is 86.5 degree at $900 \mathrm{MHz}$ and 56.3 degrees at $2.45 \mathrm{GHz}$. 


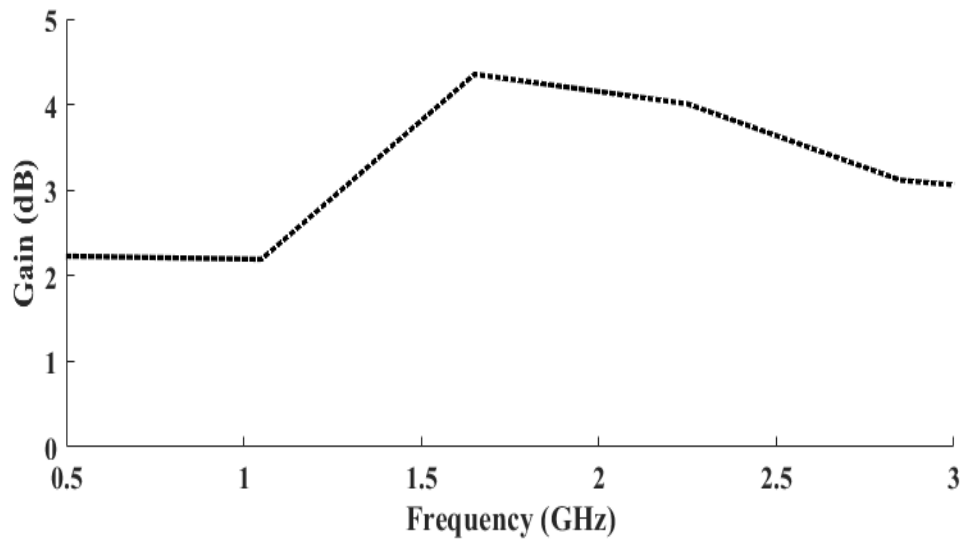

Figure 6. Simulated gain vs frequency of the proposed antenna

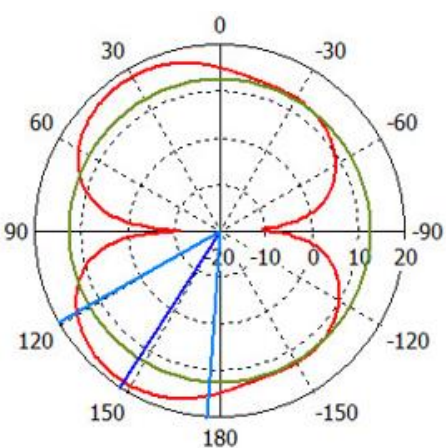

(a)

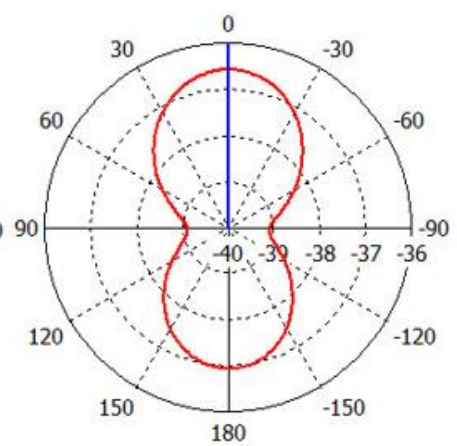

(b)

Figure 7. 2D radiation patterns of the proposed antenna at 2.45GHz (a)E plane (b) H plane

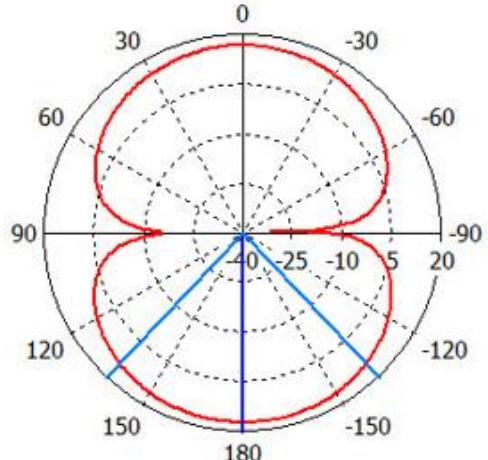

(a)

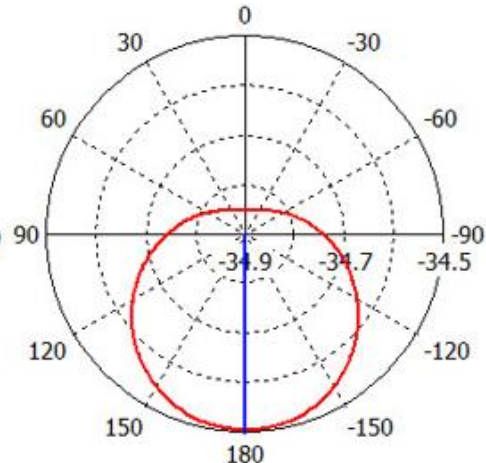

(b)

Figure 8. 2D radiation patterns of the proposed antenna at $900 \mathrm{MHz}(\mathrm{a}) \mathrm{E}$ plane (b) $\mathrm{H}$ plane

The surface current distribution of the proposed antenna for both frequencies is represented in Figure 9. It clearly observed that a maximum current is shown around the $U$ slot at $900 \mathrm{MHz}$ and around the SRRs at $2.45 \mathrm{GHz}$ and around the feed line for both frequencies. After optimisation of the antenna by CST Microwave Studio, we have launched the same antenna by HFSS. Figure 10 presents a comparison results of the reflection coefficient obtained by using CST Microwave Studio and HFSS. According to this figure, the results of the return loss obtained by CST are similar to the results computed by HFSS. The minor difference 
observed is due to the different calculation method used by both simulators. CST Microwave is based on the finite integration technique and HFSS is based on the finite element method.

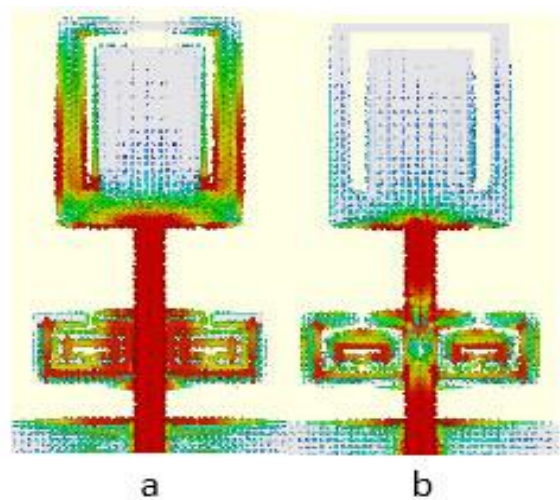

Figure 9. Surface current distributions at (a) $900 \mathrm{MHz}$, (b) $2.45 \mathrm{GHz}$

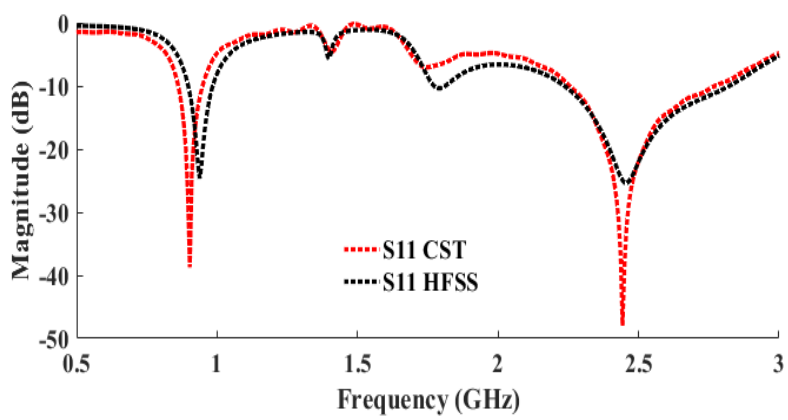

Figure 10. Comparison results between CST and HFSS

\section{Fabrication and Measurement}

After optimisation by CST microwave studio and validation of the simulated results by HFSS. we have conducted the realisation to check the performance of the simulation results of the radiation pattern and the reflection coefficient of the proposed antenna. The designed antenna was fabricated on FR4 substrate with the dielectric constant $\varepsilon r=4.4$, loss tangent tan $=0.025$ and thickness $\mathrm{h}=1.6 \mathrm{~mm}$ by using LPKF machine and tested using an R\&S VNA. the fabricated antenna is shown in Figure 11, it's having a total area of $75 \mathrm{~mm} \times 50 \mathrm{~mm}$.

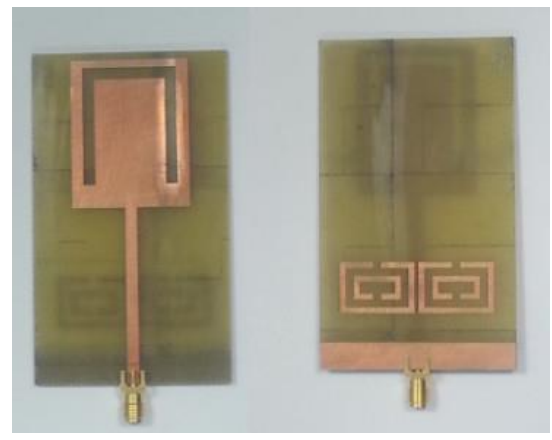

Figure 11. Photograph of the fabricated antenna 
The comparison between the measured and simulated reflection coefficient of the proposed antenna is illustrated in Figure 12. we can notice that the measured result is close to the simulated one. The difference between the measurement and simulation results is due to the error of fabrication, lower quality of SMA port used, uncertainty in substrate thickness and dielectric constant. The measured radiation pattern at $2.45 \mathrm{GHz}$ and $900 \mathrm{MHz}$ is presented in Figure 13.

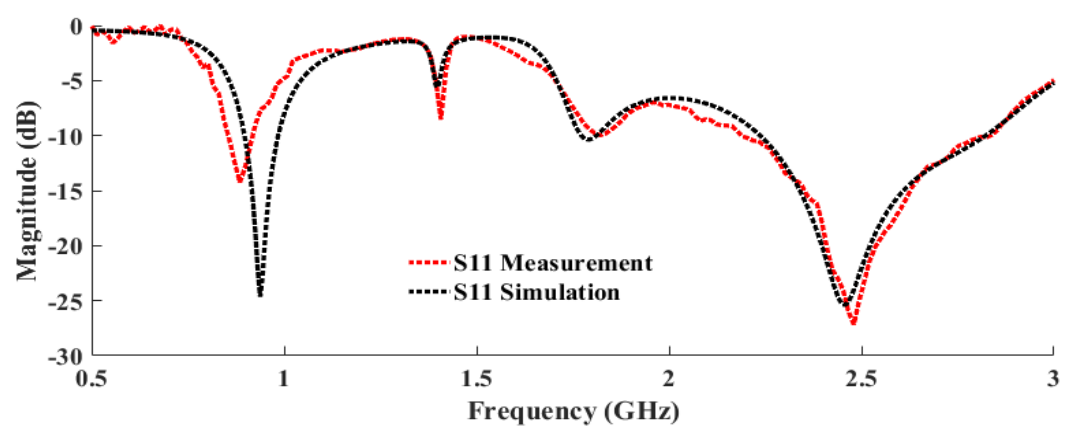

Figure 12. Comparison between simulated and measured reflection coefficients of the proposed antenna

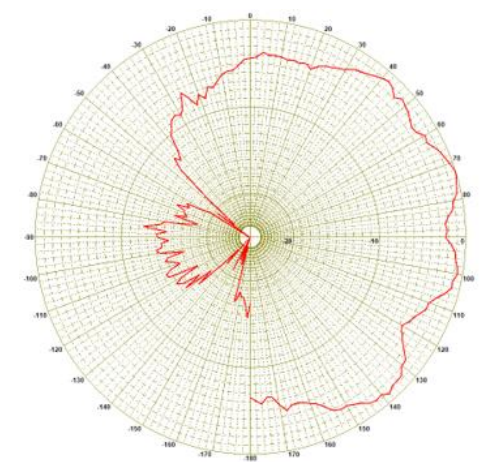

(a)

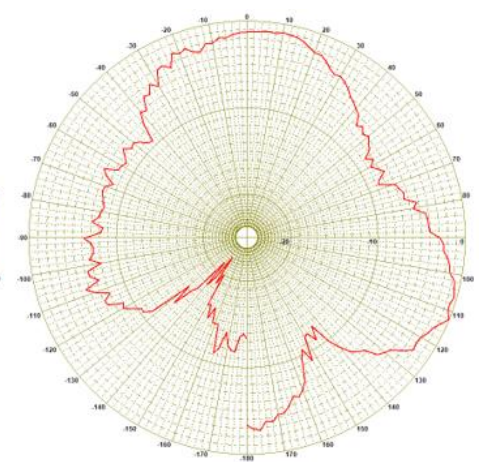

(b)

Figure 13. Measured radiation patterns at (a) $2.45 \mathrm{GHz}$ (b) $900 \mathrm{MHz}$

\section{Conclusion}

In this work, a novel design of a miniature dual-band metamaterial monopole antenna embedded $\mathrm{U}$ slot for RFID reader applications is presented. The antenna is designed, simulated and optimised by using CST microwave studio. The computed results were compared by using HFSS and a good agreement between both computed results is observed. The use of the split rings resonators makes the antenna suitable for dual-band operations and improve the antenna performance. The radiation patterns of the proposed monopole antenna are quasiomnidirectional in both $\mathrm{H}$-plane and E-plane.

\section{Acknowledgements}

We thank Mr. Mohamed LATRACH Professor in ESEO, Engineering Institute in Angers, France, for allowing us to use all the equipments and EM solvers available in his laboratory.

\section{References}

[1] Evizal, Tharek A, Sharul KA. UHF RFID Tag Antenna for Vehicle License Plate Number (e-Plate). TELKOMNIKA (Telecommunication Computing Electronics and Control). 2013; 11(2): 337-346. 
[2] Wu MY, Ke CK, Tzeng WL. Applying Context-Aware RBAC to RFID Security Management for Application in Retail Business. IEEE Asia-Pacific Services Computing Conference. 2008.

[3] Ennajih A, Zbitou J, Errkik A, Tajmouati A, El Abdellaoui L, Latrach M. A novel design of passive UHF RFID tag antenna mounted on paper. International Conference on Wireless Technologies, Embedded and Intelligent Systems (WITS), Fez, Morocco. 2017.

[4] Rao KVS, Nikitin PV, Lam SF. Antenna design for UHF RFID tags: a review and a practical application. IEEE Trans. on Antennas and Propag. 2005; 53(12): 3870-3876.

[5] Jamal MR. A Proposed Design of Unit Cell of Metamaterial for 5G Mobile Communication. TELKOMNIKA (Telecommunication Computing Electronics and Control). 2017; 15(3): 1145-1148.

[6] Veselago VG. The electrodynamics of substances with simultaneously negative values of $\varepsilon$ and $\mu$. Ph. Uspekhi. 1968; 10(4): 509-514.

[7] Pendry JB. Extremely Low Frequency Plasmons in Metallic Mesostructures. Ph. Rev. Lett. 1996; 76(25): 4773.

[8] Pendry JB, Holden AJ, Robbins DJ, Stewart WJ. Magnetism from conductors and enhanced nonlinear phenomena. IEEE Trans. on Microwave Theory and Tech. 1999; 47(11): 2075-2084.

[9] Smith DR, Padilla WJ, Vier DC, Nemat-Nasser SC, Schultz S. Composite Medium with Simultaneously Negative Permeability and Permittivity. Ph. Rev. Lett. 2000; 84(18): 4184-4187.

[10] Mookiah P, Dandekar KR. Metamaterial-Substrate Antenna Array for MIMO Communication System. IEEE Trans. on Antennas and Propag. 2009; 57(10): 3283-3292.

[11] Ennajih A, Zbitou J, Latrach M, Errkik A, El Abdellaoui L, Tajmouati A. Dual Band Metamaterial Printed Antenna Based on CSRR For RFID Applications. Int. J. of Microwave and Opt. Tech. 2017; 12(2): 106-113.

[12] Nasiri B, Errkik A, Zbitou J, Tajmouati A, El Abdellaoui L, Latrach M. A New Compact Microstrip Band-stop Filter by Using Square Split Ring Resonator. Int. J. of Microwave and Opt. Tech. 2017; 12(5): 367-373. 